\title{
Encéphalite de Rasmussen : hypothèses physiopathologiques et traitement médical
}

\section{Rasmussen's encephalitis: pathophysiological hypotheses and medical treatment}

Auteurs: Stanislas LAGARDE ${ }^{1}$

Affiliations :

1. Aix Marseille Univ, APHM, INSERM, INS, Inst Neurosci Syst, Timone Hospital, Epileptology Department, Marseille, France

Auteur Correspondant:

Dr Stanislas LAGARDE

Courriel : Stanislas.lagarde@ap-hm.fr

Adresse Postale : Service Epileptologie et Rythmologie Cérébrale, Hôpital Timone, 264 Rue Saint-Pierre, 13005, Marseille, France

Tel : 04.91.38.49.90

Fax : 04.91.38.58.26 


\section{RESUME}

L'encéphalite de Rasmussen (ER) est une maladie cérébrale inflammatoire chronique, rare et grave, qui entraîne une épilepsie résistante aux médicaments et la destruction progressive d'un hémisphère avec déficit neurologique. L'ER est associée à une détérioration de l'activité de fond EEG, une atrophie progressive à l'IRM et un hypométabolisme TEP étendu sur I'hémisphère affecté. L'ER est une maladie à médiation immunitaire, avec un rôle prédominant des cellules cytotoxiques $\mathrm{T} C D 8+$, mais également associée à une activation microgliale et à l'induction de voies complexes d'inflammasome. Le diagnostic de l'ER repose sur des critères cliniques (épilepsie réfractaire et déficit neurologique), électrophysiologiques (ralentissement unilatéral de l'EEG) et IRM (hémiatrophie). Les médicaments antiépileptiques sont généralement incapables d'arrêter les crises. Le traitement le plus efficace est I'hémisphérotomie (déconnexion chirurgicale d'un hémisphère cérébral), mais elle est associée à des déficits moteurs et neurologiques permanents. Les immunothérapies constituent une stratégie préférable au stade précoce de I'ER ou chez les patients dont la progression de la maladie est lente et/ou les déficits légers et/ou qui ne sont pas éligibles à la chirurgie. En fonction de la pathogenèse, plusieurs immunothérapies ont été essayées dans I'ER (aucune de manière exhaustive : corticostéroïde, IgIV, tacrolimus, azathioprine, adalimumab, mycophénolate mofétil, natalizumab). Cependant, seules de petites cohortes ont été rapportées sans étude comparative. Dans cet article, nous résumons quelques mécanismes physiopathologiques de I'ER, avant de rapporter les données de la littérature concernant les immunothérapies.

Keywords: focal epilepsy, drug resistant epilepsy, encephalitis, immunotherapy, neuroimmunology

Mot Clés: épilepsie focale, épilepsie pharmacorésistance, encéphalite, neuroimmunologie 


\begin{abstract}
Rasmussen's encephalitis (RE) is a rare and severe chronic inflammatory brain disease that results in drug-resistant epilepsy and progressive hemispheric destruction with neurological deficit. RE is associated with deterioration of EEG background activity, progressive atrophy on $\mathrm{MRI}$ and extensive PET hypometabolism over the affected hemisphere. RE is an immunemediated disease, with a predominant role for CD8+ cytotoxic T cells, but also associated with microglial activation and induction of complex inflammasome pathways. The diagnosis of RE is based on clinical (refractory epilepsy and neurological deficit), electrophysiological (unilateral EEG slowing) and MRI (haemiatrophy) criteria. Anti-seizure medications are generally unable to stop the seizures. The most effective treatment is hemispherotomy (surgical disconnection of one brain hemisphere), but this is associated with permanent motor and neurological deficits. Immunotherapies are a preferable strategy in the early stage of ER or in patients with slow disease progression and/or mild deficits and/or who are not eligible for surgery. Depending on the pathogenesis, several immunotherapies have been tried in RE (none exhaustively: corticosteroid, IVIG, tacrolimus, azathioprine, adalimumab, mycophenolate mofetil, natalizumab). However, only small cohorts have been reported without comparative studies. In this article, we summarise some pathophysiological mechanisms of RE, before reporting data from the literature regarding immunotherapies.
\end{abstract}




\section{Introduction}

L'encéphalite de Rasmussen (ER) est une maladie cérébrale inflammatoire chronique particulièrement grave qui entraîne une épilepsie pharmacorésistante et la destruction progressive d'un hémisphère. Ce processus inflammatoire s'accompagne d'une perte progressive des fonctions neurologiques (motrices et/ou cognitives) de l'hémisphère affecté. [1]. L'incidence annuelle est estimée à environ 2,4 cas/10 millions de personnes [1]. La maladie évolue généralement en trois phases : une phase prodromique, une phase aiguë et une phase résiduelle [1]. Le « stade prodromique » n'est pas spécifique, avec des crises épileptiques peu fréquentes et peu ou pas de déficit neurologique (par exemple, une hémiparésie légère) ou de troubles comportementaux/cognitifs. Après quelques mois, tous les patients connaissent une « phase aiguë » avec des crises fréquentes et réfractaires. Si les patients ne commencent pas un traitement approprié, ils développent, pendant la phase aiguë, un déficit neurologique progressif et irréversible (par exemple, une hémiparésie), et/ou un déclin cognitif et/ou des troubles du comportement. Une aphasie peut survenir si l'hémisphère dominant est touché. Le "stade résiduel » est marqué par des crises moins fréquentes mais des déficits neurologiques persistants et stables. La maladie touche généralement les enfants, mais $10 \%$ des cas de ER commencent chez les adolescents et les adultes [2]. L'évolution clinique de ces formes tardives est plus lente et les déficits neurologiques résiduels sont moins graves [1,2]. Dans le cas de l'ER, on observe généralement une détérioration de l'activité de fond EEG, une atrophie progressive à I'IRM et un hypométabolisme cérébral (PET-FDG) étendu à I'hémisphère affecté [1]. L'ER est une maladie à médiation immunitaire, caractérisée par une implication constante des lymphocytes T [1].

Le diagnostic de ER repose sur des caractéristiques cliniques (crises focales et déficit cortical unilatéral), électrophysiologiques (ralentissement EEG et crises unihémisphériques) et 
morphologiques (atrophie unihémisphérique +/- hypersignal), et parfois sur I'histologie (infiltration de cellules T avec activation microgliale) [3]. Cependant, en utilisant ces critères, le diagnostic précoce est souvent difficile, ce qui pose un problème majeur pour l'initiation précoce de l'immunothérapie afin de combattre la phase aiguë de la maladie et de prévenir l'apparition d'un déficit neurologique irréversible. L'analyse du LCR est anormale dans la plupart des cas (augmentation de la protéinorachie et/ou une augmentation du nombre de cellules), mais il n'existe pas de test spécifique. [4]. La présence d'une oligoclonalité n'est décrite que pour une partie du patient. Aucun auto-anticorps n'a été clairement associé à l'ER (certains ont été décrits mais avec une faible sensibilité et aucune spécificité).

Les médicaments antiépileptiques sont généralement incapables d'arrêter les crises. Le traitement curatif le plus efficace est la chirurgie basée sur des procédures d'hémisphérotomie (déconnexion chirurgicale de l'hémisphère cérébral pathologique), mais il est associé à des déficits moteurs permanents et à des déficits cognitifs [5]. Cette chirurgie reste difficile à proposer aux patients sans déficit moteur ou atteints de formes d'ER à évolution lente, non motrices et à début tardif [6]. Une approche pharmacologique avec des agents agissant sur le système immunitaire, et en particulier sur l'immunité des cellules $T$, est une stratégie préférable à un stade précoce de la maladie ou chez les patients dont la progression de la maladie est lente et les déficits légers et/ou qui ne sont pas éligibles pour une chirurgie hémisphérique.

\section{Mécanismes physiopathologiques}

Les prélèvements histologiques de patients souffrant d'ER montrent des nodules microgliaux et lymphocytaires notamment périvasculaires, une mort neuronale et une neuronophagie [7]. Les lymphocytes T cytotoxiques semblent jouer un rôle crucial car ils constituent la plupart des cellules inflammatoires observées histologiquement [8] et environ $10 \%$ de ces cellules 
sont positives au granzyme B dont certaines en étroite apposition avec des neurones confirmant leur cytotoxicité directe [8]. En outre, les cellules CD8+ peuvent également cibler les astrocytes dans les ER [9]. Dans l'ER, une activation microgliale [7] et astrocytaire [9] est aussi observée. La microglie activée, via la libération de cytokines pro-inflammatoires qui pourrait aussi participer à l'ictogenèse. En ce qui concerne l'immunité humorale, le rôle des cellules B et des auto-anticorps dans l'ER est plus controversé. Plusieurs auto-anticorps ont été trouvés dans le sérum de patients atteints d'ER, mais ils ne sont pas spécifiques de la maladie et pourraient être un phénomène secondaire dû à la disponibilité accrue d'antigènes, suite à la mort neuronale (Figure 1). Certaines observations histologiques et mécanismes physiopathologiques suspectés dans l'ER sont proches de ceux des vascularites cérébrales.

Une seule étude a réussi à reproduire les caractéristiques histologiques et cliniques de l'ER dans un modèle expérimental murin suite à la transplantation des cellules mononucléaires du sang périphérique de patients atteints de ER chez des souris immunodéficientes, confirmant le rôle de l'auto-immunité à médiation cellulaire de cette maladie [10]. Les auteurs ont pu tester l'effet de deux immunothérapies dans leur modèle : Immnuglobulines intraveineuses (IgIV) et natalizumab, à deux moments, précoce ou tardif. Les injections précoces d'IgIV et de natalizumab ont permis une diminution de la fréquence des crises, et de l'infiltration inflammatoire cérébrale. Par contre, l'injection tardive de natalizumab si elle a pu limiter l'infiltration inflammatoire cérébrale, n'a pas eu d'effet sur les crises. Cela suggère qu'une fois la maladie établie, la réduction de l'infiltration des cellules $T$ dans le cerveau n'est pas suffisante en soi pour réduire la fréquence des crises.

Dans l'ensemble, ces données confirment le bien-fondé de l'immunothérapie dans l'ER, et en particulier des traitements ciblant l'immunité des lymphocytes T. Les données suggèrent une fenêtre thérapeutique avec un potentiel bénéfique plus élevé au début de la maladie, lorsque 
les dommages aux neurones et aux astrocytes sont limités et que l'inflammation cérébrale pourrait être inversée [11]. Cependant, la persistance de l'auto-immunité et de l'inflammation des cellules $\mathrm{T}$ à un stade ultérieur de la maladie plaide en faveur de l'intérêt de l'immunothérapie, même à un stade plus tardif chez les patients ne pouvant pas être opérés.

\section{Principes généraux de la prise en charge de l'encéphalite de Rasmussen}

Les antiépileptiques ont une efficacité limitée dans l'ER et la chirurgie représente la meilleure option pour contrôler les crises et arrêter la détérioration neurologique [1]. Les techniques chirurgicales visent à obtenir une déconnexion fonctionnelle de l'hémisphère affecté avec des conséquences fonctionnelles inévitables (hémianopsie homonyme et hémiplégie notamment). La récupération de la marche indépendante est attendue, mais pas la motricité fine de la main. Les immunothérapies peuvent-être utilisées dans l'ER au stade précoce, ou en cas de balance bénéfice-risque défavorable de la chirurgie (ex : faible déficit neurologique, forme tardive, lentement progressive, hémisphère dominant) (Figure 2). Par contre, chez les patients dont la balance bénéfices-risques est favorable à la chirurgie, l'essai d'immunothérapie ne doit pas retarder la chirurgie.

\section{Données relatives au traitement médical}

\subsection{Première ligne}

Les corticostérö̈des agissent via des effets génomiques et non génomiques sur les réponses immunitaires innées et adaptatives, avec une réduction de la transcription de plusieurs gènes pro-inflammatoires, une inhibition des cellules présentatrices d'antigènes, une augmentation de l'apoptose des lymphocytes T et un basculement de la réponse immunitaire du phénotype Th1 au phénotype Th2 [12]. Leur utilisation à long terme est limitée par la fréquence des effets secondaires : syndrome de Cushing, ostéoporose, hypertension et infections. Dans l'ER, globalement, environ $70 \%$ (83/117) des patients répondent initialement aux corticostéroïdes 
[13]. Cependant, certaines études ont suggéré une perte d'efficacité à long terme [14] et le taux d'effets secondaires à long terme/interruption du traitement était extrêmement élevé [14]. Ainsi, les corticostéroïdes semblent être un traitement très efficace en première intention, mais ils doivent être suivis d'un autre traitement à long terme pour limiter leurs effets secondaires.

D'autres études ont rapporté I'utilisation des immunoglobulines intraveineuses (IgIV) dans I'ER. Les IgIV agissent sur l'inflammation médiée par les anticorps, qui ne semble pas être cruciale dans la pathogenèse de l'ER, mais aussi par la modulation des lymphocytes T (ce qui pourrait mieux expliquer leur efficacité dans I'ER) [15]. L'efficacité des IgIV pour réduire les crises (globalement $30 \%$ dans les séries de plus de 5 patients), semble être inférieure à celle des corticostéroïdes. Les plasmaphérèses réduisent les taux d'anticorps et d'autres médiateurs immunitaires et les fractions du complément. Les données existantes sont principalement des rapports de cas isolés avec un effet initial positif mais une récurrence à long terme [13]. Cependant, dans les séries plus importantes, les taux de réponse aux crises étaient faibles 2/13 [13], et l'impact sur le déficit neurologique et cognitif limité.

Il semble donc logique de proposer les corticostéroïdes en première intention et de réserver les immunoglobulines / plasmaphérèses en cas d'échec et avant de commencer un autre traitement au long cours (Figure 3). Cela n'exclut pas leur utilisation à long terme, au cas par cas, chez les patients avec une très bonne réponse (malgré le caractère chronophage de leur administration régulière).

\subsection{Inhibiteur des lymphocytes}

L'un des premiers traitements étudiés ciblant spécifiquement les lymphocytes a été le tacrolimus. Il a montré une efficacité variable mais plutôt faible sur les crises d'épilepsie dans les séries [16,17]. Cependant, il a ralenti l'aggravation neurologique et l'atrophie IRM 
progressive par rapport à une cohorte historique non traitée. [16]. Par rapport au traitement par IgIV, I'utilisation du tacrolimus a été associée à une réponse clinique comparable, mais à un taux plus élevé d'effets indésirables. [16]. Une étude rétrospective a rapporté l'utilisation d'azathioprine dans une cohorte de 53 enfants atteints de ER en relais de la corticothérapie initiale [18]. $89 \%$ des patients ont présenté une réponse soutenue aux crises (Figure 3). Par rapport au groupe non traité par l'azathioprine, le groupe traité a eu un délai plus long avant l'apparition de l'épilepsie partielle continue, de l'hémiparésie et la réalisation de l'hémisphérotomie (mais sans différence significative en termes de prévalence d'apparition de ces événements entre les deux groupes). L'azathioprine n'a pas eu d'effet significatif sur la progression de l'atrophie en IRM, ni sur l'évolution cognitive. Le mycophénolate mofétil a été utilisé dans certains rapports de cas : avec un effet potentiel sur les crises et le déficit neurologique [13]. II est généralement mieux toléré que l’azathioprine.

\subsection{Autres immunothérapies}

Suite à l'observation de l'augmentation du TNF $\alpha$ chez certains patients avec ER et de son implication dans l'ictogenèse [4], nous avons commencé à utiliser les anti-TNF $\alpha$ (adalimumab) chez 11 patients [19]. Nous avons trouvé 5/11 patients répondeurs en termes de crise (Figure 3) et 3 de ces 5 avec une stabilité cognitive et une amélioration motrice. Le suivi à long terme a montré une bonne tolérance. L'efficacité semblait meilleure chez les patients ayant un début tardif et une maladie lentement progressive.

Bien que la justification physiopathologique de l'utilisation du rituximab ne semble pas évidente en raison de son effet principalement sur les lymphocytes B, il pourrait agir comme un traitement indirectement anti-lymphocytes T. [20]. Certains rapports de cas ont suggéré un effet bénéfique, mais d'autres n'ont trouvé qu'un effet transitoire ou aucun effet (Figure 
3) [13]. Ainsi, les données sont encore incohérentes pour donner une conclusion claire sur son intérêt.

Le natalizumab est un anticorps qui inhibe la migration des cellules immunitaires à travers la barrière hémato-encéphalique. D'un point de vue physiopathologique, son utilisation dans I'ER semble intéressante, notamment dans la phase précoce de la maladie, afin d'éviter l'invasion du cerveau par les lymphocytes T [10]. Un rapport de cas a montré une efficacité sur l'épilepsie d'un patient, mais son évolution neurologique n'a pas été décrite [21].

Enfin d'autres molécules ont été utilisés mais dans un nombre limité de rapport de cas (cyclophosphamide, mitoxantrone, anakinra, alemtuzumab, methotrexate intrathecal, ciclosporine, thalidomide, basilixumab, antiviraux, interferon alpha, transplantation de cellules souches) [13]. II est difficile d'extrapoler ces résultats qui sont soumis à un fort biais de publication.

\section{Conclusion}

Le traitement médical de l'ER repose sur des traitements antiépileptiques et antiinflammatoires/immunomodulateurs. Ceux-ci ne doivent pas retarder I'hémisphérotomie lorsqu'elle est indiquée, en raison d'une possible meilleure efficacité et d'un meilleur devenir cognitif lorsqu'elle est réalisée à un stade précoce. Les immunothérapies sont cependant intéressantes à un stade précoce de la maladie ou en cas de contre-indication/non-indication à I'hémisphérotomie (forme lente, sans déficit neurologique marqué, touchant l'hémisphère dominant, début tardif). On privilégiera en première intention les corticostéroïdes (IV ou oraux). En relais/seconde ligne, les données d'efficacité les plus robustes concernent l'azathioprine, l'adalimumab et le rituximab. Le tacrolimus n'a que peu d'effet sur les crises bien qu'il ralentisse la progression de l'atrophie et du déficit neurologique 


\section{Déclaration d'intérêt :}

L'auteur ne déclare aucun conflit d'intérêt en rapport avec cet article.

\section{REFERENCES}

[1] Varadkar S, Bien CG, Kruse CA, Jensen FE, Bauer J, Pardo CA, et al. Rasmussen's encephalitis: Clinical features, pathobiology, and treatment advances. Lancet Neurol 2014;13:195-205. https://doi.org/10.1016/S1474-4422(13)70260-6.

[2] Dupont S, Gales A, Sammey S, Vidailhet M, Lambrecq V. Late-onset Rasmussen Encephalitis: A literature appraisal. Autoimmun Rev 2017;16:803-10. https://doi.org/10.1016/j.autrev.2017.05.022.

[3] Bien CG, Granata T, Antozzi C, Cross JH, Dulac O, Kurthen M, et al. Pathogenesis, diagnosis and treatment of Rasmussen encephalitis: A European consensus statement. Brain 2005;128:454-71. https://doi.org/10.1093/brain/awh415.

[4] Takahashi Y, Mine J, Kubota Y, Yamazaki E, Fujiwara T. A substantial number of Rasmussen syndrome patients have increased IgG, CD4+ T cells, TNF $\alpha$, and Granzyme B in CSF. Epilepsia 2009;50:1419-31. https://doi.org/10.1111/j.15281167.2008.01977.x.

[5] Greicius MD, Flores BH, Menon V, Glover GH, Solvason HB, Kenna H, et al. RestingState Functional Connectivity in Major Depression: Abnormally Increased Contributions from Subgenual Cingulate Cortex and Thalamus. Biol Psychiatry 2007;62:429-37. https://doi.org/10.1016/j.biopsych.2006.09.020.

[6] Harris WB, Westley Phillips H, Chen JS, Weil AG, Ibrahim GM, Fallah A. Seizure outcomes in children with Rasmussen's encephalitis undergoing resective or hemispheric epilepsy surgery: An individual participant data meta-analysis. J Neurosurg Pediatr 2020;25:274-83. https://doi.org/10.3171/2019.9.PEDS19380.

[7] Pardo CA, Vining EPG, Guo L, Skolasky RL, Carson BS, Freeman JM. The Pathology of Rasmussen Syndrome: Stages of Cortical Involvement and Neuropathological Studies in 45 Hemispherectomies. Epilepsia 2004;45:516-26. https://doi.org/10.1111/J.00139580.2004.33103.X.

[8] Bien CG, Bauer J, Deckwerth TL, Wiendl H, Deckert M, Wiestler OD, et al. Destruction of neurons by cytotoxic T cells: A new pathogenic mechanism in Rasmussen's encephalitis. Ann Neurol 2002;51:311-8. https://doi.org/10.1002/ana.10100.

[9] Bauer J, Elger CE, Hans VH, Schramm J, Urbach H, Lassmann H, et al. Astrocytes are a specific immunological target in Rasmussen's encephalitis. Ann Neurol 2007;62:6780. https://doi.org/10.1002/ana.21148.

[10] Kebir H, Carmant L, Fontaine F, Béland K, Bosoi CM, Sanon NT, et al. Humanized mouse model of Rasmussen's encephalitis supports the immune-mediated hypothesis. J Clin Invest 2018;128:2000-9. https://doi.org/10.1172/JCI97098.

[11] Liba Z, Muthaffar O, Tang J, Minassian B, Halliday W, Branson H, et al. Rasmussen encephalitis: Response to early immunotherapy in a case of immune-mediated encephalitis. Neurol Neuroimmunol Neurolnflammation 2015;2:e69. https://doi.org/10.1212/NXI.0000000000000069.

[12] Vandewalle J, Luypaert A, De Bosscher K, Libert C. Therapeutic Mechanisms of Glucocorticoids. Trends Endocrinol Metab 2018;29:42-54.

https://doi.org/10.1016/j.tem.2017.10.010. 
[13] Orsini A, Foiadelli T, Carli N, Costagliola G, Masini B, Bonuccelli A, et al. Rasmussen ' s encephalitis : From immune pathogenesis towards targeted- therapy. Seizure Eur J Epilepsy 2020;81:76-83.

[14] Bahi-Buisson N, Villanueva V, Bulteau C, Delalande O, Dulac O, Chiron C, et al. Long term response to steroid therapy in Rasmussen encephalitis. Seizure 2007;16:485-92. https://doi.org/10.1016/j.seizure.2007.03.002.

[15] Lünemann JD, Nimmerjahn F, Dalakas MC. Intravenous immunoglobulin in neurology-mode of action and clinical efficacy. Nat Rev Neurol 2015;11:80-9. https://doi.org/10.1038/nrneurol.2014.253.

[16] Bien CG, Tiemeier H, Sassen R, Kuczaty S, Urbach H, Von Lehe M, et al. Rasmussen encephalitis: Incidence and course under randomized therapy with tacrolimus or intravenous immunoglobulins. Epilepsia 2013;54:543-50. https://doi.org/10.1111/epi.12042.

[17] Takahashi Y, Yamazaki E, Mine J, Kubota Y, Imai K, Mogami Y, et al. Immunomodulatory therapy versus surgery for Rasmussen syndrome in early childhood. Brain Dev 2013;35:778-85. https://doi.org/10.1016/j.braindev.2013.01.010.

[18] Pellegrin S, Baldeweg T, Pujar S, D’Arco F, Cantalupo G, Varadkar S, et al. Immunomodulation with Azathioprine therapy in Rasmussen syndrome: A multimodal evaluation. Neurology 2020;96:10.1212/WNL.0000000000011004. https://doi.org/10.1212/wnl.0000000000011004.

[19] Lagarde S, Villeneuve N, Trébuchon A, Kaphan E, Lepine A, McGonigal A, et al. Antitumor necrosis factor alpha therapy (adalimumab) in Rasmussen's encephalitis: An open pilot study. Epilepsia 2016;57:956-66. https://doi.org/10.1111/epi.13387.

[20] Liossis SNC, Sfikakis PP. Rituximab-induced B cell depletion in autoimmune diseases: Potential effects on T cells. Clin Immunol 2008;127:280-5. https://doi.org/10.1016/j.clim.2008.01.011.

[21] Bittner S, Simon OJ, Gobel K, Bien CG, Meuth SG, Wiendl H. Rasmussen encephalitis treated with natalizumab. Neurology 2013;81:395-7. https://doi.org/10.1212/wnl.0b013e31829c5ceb. 


\section{Points Essentiels}

1. L'encéphalite de Rasmussen se présente avec : un ralentissement de l'activité de fond EEG, une épilepsie pharmacorésistante à point de départ unihémipsherique, une hémiatrophie cérébrale et des déficits neurologiques unilatéraux.

2. Sa physiopathologie implique une infiltration cérébrale par des lymphocytes activés, essentiellement CD8+ ; une activation microgliale et d'une cascade pro-inflammatoire.

3. Le traitement de référence est l'hémispherotomie chirurgicale, mais avec des conséquences fonctionnelles inévitables.

4. A la phase aiguë de la maladie ou chez les patients sans possibilité chirurgicale (déficit neurologique modéré, début tardif, forme lente, atteinte de l'hémisphère dominant), les immunothérapies sont utiles.

5. En première ligne le traitement le plus efficace est la corticothérapie.

6. En relais de celle-ci, on peut proposer des traitements ciblant les lymphocytes $T$ (ex: azathioprine) ou d'autres voies inflammatoires (ex : anti-TNF alpha)

\section{Légendes des figures}

Figure 1 montrant les principaux mécanismes physiopathologiques observés dans I'ER avec le spectre d'action des immunothérapies les plus fréquemment utilisées. (Illustrations de smart.servier.com)

Figure 2 illustrant les trois phases et les diverses formes de la maladie; et montrant les fenêtres thérapeutiques / les indications pour le traitement par immunothérapies.

Figure 3 : Résumé de l'efficacité des immunothérapies avec plus de 10 patients rapportés dans la littérature, concernant les crises d'épilepsie (critères de réponse variables selon les études). 